\title{
Treadmill exercise activates ATF3 and ERK1/2 downstream molecules to facilitate axonal regrowth after sciatic nerve injury
}

\author{
Ji-Eun Kim, Yeong-Hyun Cho, Tae-Beom Seo* \\ Department of Kinesiology, College of Natural Science, Jeju National University, Jeju, Korea
}

The purpose of this study was to investigate the effect of treadmill exercise on activating transcription factors such as activating transcription factor 3 (ATF3) and extracellular signal-regulated kinase (ERK1/2) signaling pathway to facilitate axonal regrowth after sciatic nerve injury (SNI). The experimental rats divided into the normal control $(n=10)$, sedentary groups for $7(n=10)$ and 14 days $(n=10)$ post crush, exercise group for $7(n=10)$ and 14 days $(n=10)$ post crush (dpc). The rats in exercise groups run on treadmill device at a speed of $8 \mathrm{~m} / \mathrm{min}$ for $20 \mathrm{~min}$ once a day according to exercise duration. In order to evaluate specific regeneration markers and axonal elongation in injured sciatic nerve, we applied immunofluorescence staining and western blot techniques. Treadmill exercise further increased growth-associated protein (GAP43) expression and axonal regrowth at 7 and $14 \mathrm{dpc}$ than those in sedentary group. Among mitogen-activated protein kinase downstream molecules, phospho-ERK1/2 (p-ERK1/2) was enhanced by treadmill exercise at only $7 \mathrm{dpc}$ and decreased to basal level 14 days later. But c-Jun N-terminal kinase, c-Jun, and phospho-cyclic adenosine monophosphate response element-binding protein showed a tendency to increase continuously until $14 \mathrm{dpc}$ by exercise. ATF3 expression in exercise group was upregulated at both 7 and $14 \mathrm{dpc}$ compared to the sedentary group. These results indicate that treadmill exercise had beneficial effect on expression of regeneration-related proteins after SNI, suggesting that exercise might be one of various therapeutic strategies for sciatic nerve regeneration.

Keywords: Sciatic nerve, Regeneration, Axonal regrowth, Treadmill exercise, Activating transcription factor 3, Mitogen-activated protein kinase

\section{INTRODUCTION}

Peripheral nervous system (PNS) axons can voluntary regenerate the injured axons, but central nervous system (CNS) axons cannot. This reason is because Schwann cells in PNS afford essential support by regenerative response of cellular and molecular changes in contrast to the oligodendrocytes and astrocytes in CNS (Gordon, 2016). But some previous studies suggested that spontaneous axonal regrowth after sciatic nerve injury (SNI) not only takes a long time to achieve a satisfied functional recovery but also does not lead to a complete recovery (Park and Hoke, 2014). In general, the distal stump of the injured sciatic nerves undergoes Wallerian degeneration (Arthur-Farraj et al., 2012; Grinsell and

Keating, 2014), which Schwann cells and myelin break down and macrophages infiltrate around the injury area.

The regeneration process of the injured sciatic nerve has been well documented in human and animal experiments (Ko et al., 2018; Wanner et al., 2017). Specifically, Schwann cell proliferation is generated at the early stage of the regeneration and proliferating cells migrate toward the distal region to the injury site by releasing neurotrophic factors. Then migrating Schwann cells differentiate for re-myelination. Finally, regeneration is finished by nerve innervation on target muscle (Glenn and Talbot, 2013), representing an important role of Schwann cells in sciatic nerve regeneration.

Schwann cell proliferation after SNI is regulated by genes asso-

${ }^{*}$ Corresponding author: Tae-Beom Seo (iD https://orcid.org/0000-0001-9213-2251 Department of Kinesiology, College of Natural Science, Jeju National University, 102 Jejudaehak-ro, Jeju 63243, Korea

E-mail: seotb@jejunu.ac.kr

Received: February 24, 2020 / Accepted: March 27, 2020 
ciated with the cell cycle and cell division, and these cell biological changes facilitate axonal regeneration of the injured peripheral nerves (Jiang et al., 2017). In previous studies on cell proliferation and nerve regeneration, cell division cycle- $2(\mathrm{Cdc} 2$ or $\mathrm{Cdk} 1)$ promoting G2-M phase transition in the cell cycle has been demonstrated to enhance axonal elongation of the injured sciatic nerve by activating Schwann cell proliferation and neurotrophic factors including brain-derived neurotrophic factor (Han et al., 2007) as well as ERK1/2 are reported to be activated by SNI to enhance Schwann cell proliferation (Seo et al., 2009). In addition, ATF3 or CREB for activating regeneration associated genes (RAGs) are upregulated in cell body of injured peripheral nerve. It has been known that these RAGs are increased by inhibition of free oxygen radicals (Kalender et al., 2009), cell transplantation therapy (Chen et al., 2007) and physical activity (Gómez-Pinilla et al., 2002) after SNI.

Various physical activities including treadmill, swimming, resistance, and wheel running exercise have frequently recommended as a therapeutic modality for improving histological changes and functional recovery after SNI (Armada-da-Silva et al., 2013). According to related studies, treadmill training stimulates proliferation of Schwann cells that support axonal regeneration at early stage of sciatic nerve regeneration (Seo et al., 2009), and physical activity improves the capacity of axonal growth and re-innervation with target muscles (English et al., 2009; Udina et al., 2011a).

With these results mentioned by previous studies, physical activity is essential for axonal regeneration and biochemical alteration after SNI. However the rationale of treadmill exercise on activation of ATF3 and ERK1/2 signaling pathway at early stage of sciatic nerve regeneration is still not clear. Therefore, the purpose of this study was to investigate effect of treadmill training on axonal regrowth at 7 and 14 days after SNI through activation of ATF3 and ERK1/2 downstream molecules.

\section{MATERIALS AND METHODS}

\section{Experimental animals}

Male Sprague-Dawley rats (160 g, 6 weeks old) were used in this experiment. They were allocated into 5 groups: the normal control $(n=10)$, sedentary groups for $7(n=10)$ and 14 days $(n=10)$ postinjury, exercise group for $7(n=10)$ and 14 days $(n=10)$ postinjury. Animals were maintained at a constant room temperature of $22^{\circ} \mathrm{C}$ and $60 \%$ of humidity under 12/12-hr light-dark cycle. They were accepted to eat commercial rat chow (Samyang Co., Seoul, Korea) and water ad libitum. The experimental procedures must be approved by the ethics committee of Jeju National University and followed animal experiment guide in our institution before this study.

\section{Sciatic nerve injury}

For the surgical procedures, the rats were anesthetized with using an animal inhalation narcosis control (Jeungdo Bio \& Plant, Seoul, Korea). First of all, the rats were placed into the chamber with $2 \%-2.5 \%$ concentration of isoflurane for anesthesia and then $1.5 \%-1.8 \%$ concentration for maintenance during SNI. The left sciatic nerve was crushed with a pair of forceps for $1 \mathrm{~min}$ and 30 sec at intervals (Seo et al., 2006). After surgery, anesthetized animals were then placed on a heating pad maintained at $37^{\circ} \mathrm{C}$, and then they were put in their cages for resting. All rats were sacrificed 7 and 14 days later.

\section{Treadmill exercise}

The experimental rats were adapted to treadmill exercise about $10 \mathrm{~min}$ for a week before starting the experiment. Sciatic nerve surgery was performed to exercise and sedentary groups but rats in control group were not crushed. The animals had rest for 2 days after sciatic crush injury. Treadmill exercise was conducted on third postoperative day. The animals in exercise group ran on the treadmill device (Jeungdo Bio \& Plant) at $8 \mathrm{~m} / \mathrm{min}$ for $20 \mathrm{~min}$ with no inclination according to exercise period.

\section{Western blot analysis}

The dissected sciatic nerves were rinsed with phosphate-buffered saline (PBS), and lysed in Triton lysis buffer. Being denatured proteins were separated on sodium dodecyl sulfate-polyacrylamide gel and then transferred onto polyvinylidene difuoride membrane on ice at $200 \mathrm{~mA}$ for $2 \mathrm{hr}$. The membranes were blocked in TBS including 5\% skim milk, $0.1 \%$ Tween 20 . Then, the membranes were incubated overnight with primary antibodies at $4^{\circ} \mathrm{C}$. Protein $(20 \mu \mathrm{g})$ was used for western blot analysis using anti-GAP-43 mouse monoclonal antibody (1:1,000, Santa Cruz Biotechnology, Santa Cruz, CA, USA), antiphosphorylated ERK1/2 rabbit polyclonal antibody (1:2,000, Cell Signaling Biotechnology, Danvers, MA, USA), antiphosphorylated CREB rabbit monoclonal antibody (1:1,200, Cell Signaling Biotechnology), anti-p38MAPK rabbit polyclonal antibody (1:700, Cell Signaling Biotechnology), anti-ATF3 rabbit polyclonal antibody (1:700, Santa Cruz Biotechnology), antiphosphorylated c-Jun rabbit polyclonal antibody (1:1,200, Cell Signaling Biotechnology), anti-c-Jun N-terminal kinase (JNK) rabbit polyclonal antibody (1:1,200, Cell Signaling 
Biotechnology), anti- $\beta$-actin mouse monoclonal antibody (1:2,000, Santa Cruz Biotechnology), and goat anti-mouse or goat anti-rabbit horseradish peroxidase conjugated secondary antibody (1:1,000, GeneTex Inc., Irvine, CA, USA) were used. The blotting proteins were detected by using Westar enhanced cheminescence substrates (Cyanagen, Bologna, Italy). Analysis of protein density was performed using Chemidoc (Bio-Rad, Hercules, CA, USA).

\section{Immunofluorescence staining}

Nerve segments were longitudinally sliced on a cryostat at $-20^{\circ} \mathrm{C}$. For immunofluorescence staining, sections were incubated in PBS with $4 \%$ paraformaldehyde and $4 \%$ sucrose for $40 \mathrm{~min}$ and rinsed 3 times. $0.5 \%$ NP-40 in PBS was treated for cell permeability and $2.5 \%$ horse serum and $2.5 \%$ bovine serum albumin were treated for inhibition of background for $4 \mathrm{hr}$ at room temperature. The following primary antibodies were used for immunostaining: anti-GAP-43 (1:1,000, Santa Cruz Biotechnology) and anti-neurofilament-200 (NF-200) rabbit polyclonal antibody (1:700, Sigma-Aldrich, Darmstadt, Germany) antibodies. Then they were incubated with rhodamine-goat anti-rabbit secondary antibody (1:600, Molecular Probes, Eugene, OR, USA) or fluorescein-goat anti-mouse antibody (1:500, Molecular Probes) for $90 \mathrm{~min}$ at room temperature. The sections were viewed with a Nikon fluorescence microscope (Nikon model E-600, Nikon, Kawasaki, Japan), and the images were analyzed using Adobe Photoshop (Adobe CS6, San Jose, CA, USA).

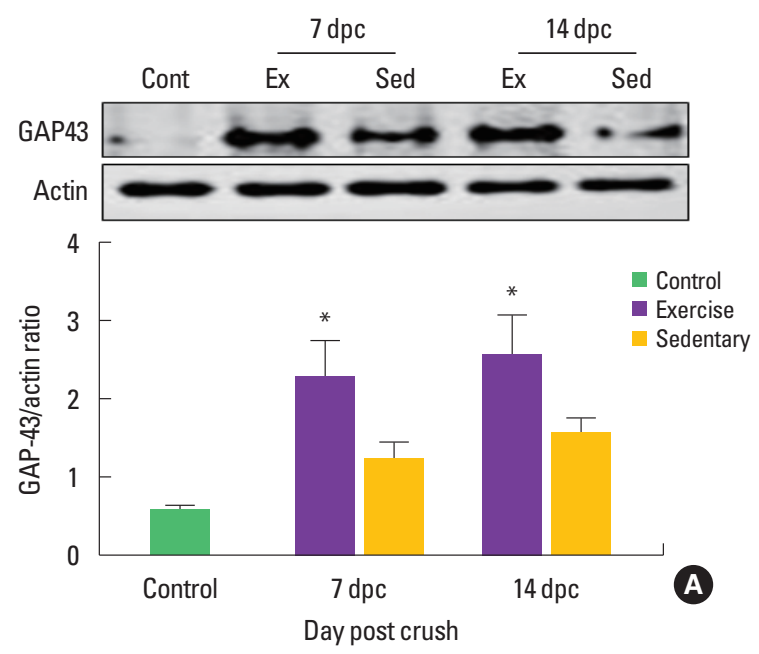

\section{Statistical analysis}

All the data is presented as a mean \pm standard error. Statistical analysis was performed using one-way analysis of variance followed by Duncan post boc test. The significance level was set at $P<0.05$. All graphs were shown by using Prism 6 (GraphPad, La Jolla, CA, USA).

\section{RESULTS}

Treadmill exercise increases GAP-43 and axonal elongation in the distal area of the injured sciatic nerve at 7 and $14 \mathrm{dpc}$

To investigate the effect of treadmill exercise on axonal regrowth, we analyzed time-dependent changes in GAP- 43 known as a specific marker for peripheral nerve regeneration. GAP-43 was expressed at 7 and 14 after SNI, and treadmill exercise significantly increased GAP-43 induction at all time points compared to those in the sedentary group (Fig. 1A). At $7 \mathrm{dpc}$, the immunohistochemistry images in $5 \mathrm{~mm}$ distal to the damage site showed increased axonal elongation stained with NF-200. And colocalization of GAP-43 with NF-200 was higher in treadmill group than those seen in the sedentary group (Fig. 1B).

\section{Treadmill exercise upregulates activation of ERK $1 / 2$ and CREB signaling pathway at early stage of sciatic nerve regeneration}

To examine the possible link between mitogen-activated protein
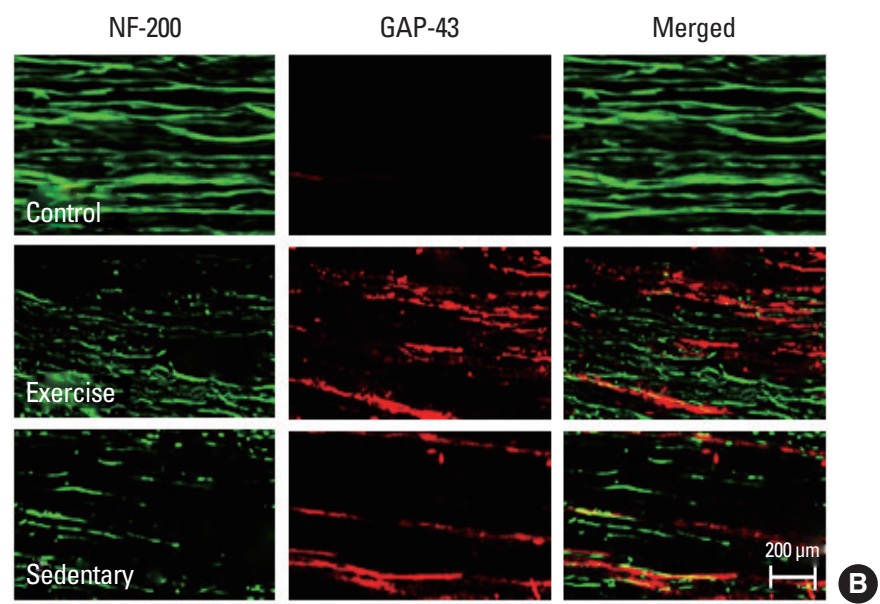

Fig. 1. Treadmill exercise increased GAP-43 expression levels and axonal elongation in the injured sciatic nerve. (A) GAP-43 was expressed in the injured sciatic nerve at 7 and 14 days post crush (dpc) and was further upregulated by treadmill exercise. In the quantitative graph, GAP-43 in exercise group (Ex) was significantly enhanced at each time point compared to sedentary group (Sed). (B) Immunofluorescence images of NF-200 and GAP-43 proteins in $5 \mathrm{~mm}$ distal to the injury site at $7 \mathrm{dpc}$. The GAP-43 signals generally overlapped with NF-200-labeled axons, and were higher in Ex than Sed. ${ }^{*} P<0.05$ vs. the control group. dpc, days post crush; Cont, control; Ex, treadmill exercise; Sed, sedentary. 

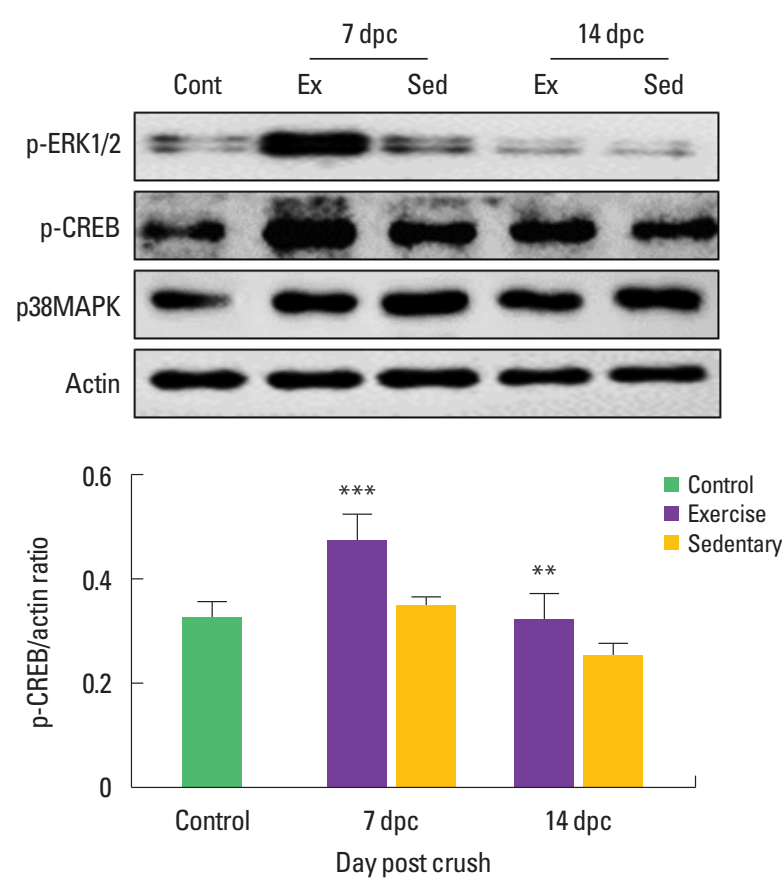
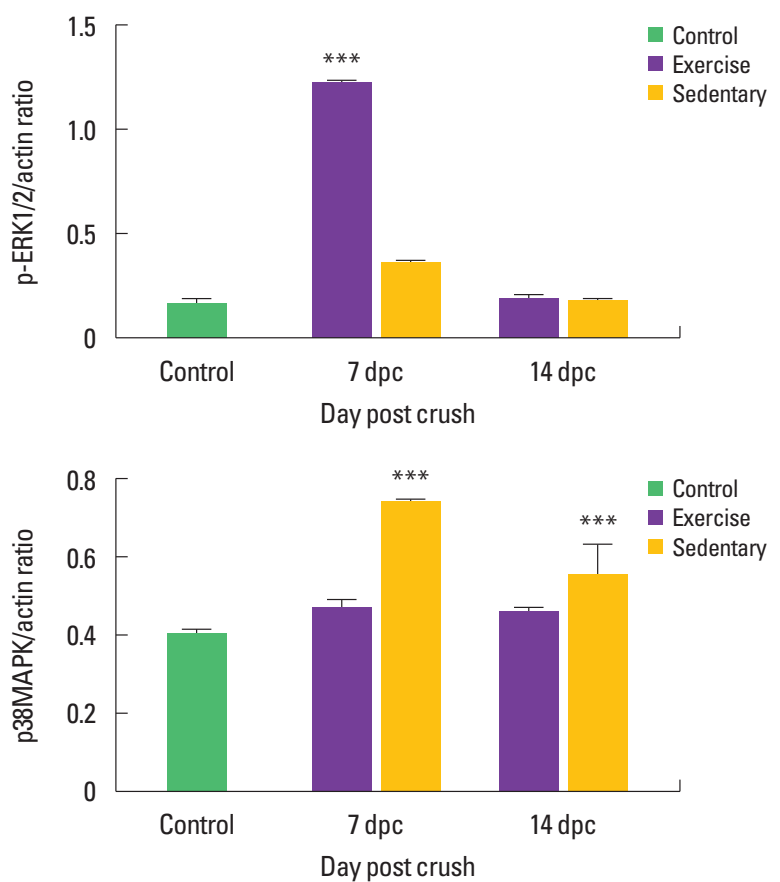

Fig. 2. Treadmill exercise upregulated expression levels of ERK1/2 and CREB at early stage of sciatic nerve regeneration. (A) p-ERK1/2 protein was increased in exercise group (Ex) at $7 \mathrm{dpc}$, and then it was dramatically decreased at $14 \mathrm{dpc}$. Treadmill exercise significantly upregulated p-CREB expression levels at both 7 and $14 \mathrm{dpc}$. But p38MAPK was further decreased in exercise group than those in the sedentary group (Sed). (B) The lower panel shows quantitation of western blot analysis. ${ }^{* *} P<0.01,{ }^{* * *} P<0.001$ vs. the control group. dpc, days post crush; Cont, control; Ex, treadmill exercise; Sed, sedentary.

kinase (MAPK) and phospho-CREB (p-CREB), we identified time-dependent alterations in phosphorylated ERK1/2 and CREB in the injured sciatic nerves. As shown in Fig. 2. Phospho-ERK1/2 expression levels were transiently increased at $7 \mathrm{dpc}$, and then decreased by a basal level at $14 \mathrm{dpc}$. At $7 \mathrm{dpc}$, treadmill exercise significantly increased p-ERK1/2 activity compared to those in the sedentary group. Phospho-CREB didn't showed significant differences between control and sedentary groups, but in treadmill exercise group, $\mathrm{p}-\mathrm{CREB}$ expression levels were significantly increased compared to the sedentary group. Moreover, expression levels of p38MAPK, a negative regulator of Schwann cell differentiation, were significantly decreased in the treadmill exercise group when compared to the sedentary group.

\section{Treadmill exercise increases phosphorylation of ATF3-mediated c-Jun at early stage of sciatic nerve regeneration}

To examine proliferating Schwann cell and axonal elongation after SNI, we examined time-dependent changes in ATF3, JNK, and p-c-Jun levels in the damaged sciatic nerves. As shown in Fig. 3. ATF3 expression levels were further increased at only 14 dpc in the sedentary than the control group, but treadmill exer- cise significantly increased induction levels of ATF3 at 7 and 14 dpc compared to those in the sedentary group. At $14 \mathrm{dpc}$, JNK protein levels were significantly upregulated in the exercise group. In addition, phosphorylated c-Jun levels showed significant differences in the exercise group than those in the control and sedentary group at 7 and $14 \mathrm{dpc}$.

\section{DISCUSSION}

Recent studies have shown that performing regular exercise can improve somatosensory functions after peripheral nerve injury (Martínez de Albornoz et al., 2011; Teodori et al., 2011). But these studies have limitations in reporting the exact biochemical mechanisms of the stimulation caused by physical activity. Our data suggest that GAP-43 protein activated by treadmill exercise may contribute to promoting axonal elongation at 7 and 14 days after SNI. GAP-43 has been known as RAGs accumulated regeneration proteins that stimulate axonal sprouting from proximal to distal stump of the injured sciatic nerve (Moore and Goldberg, 2011). In particular, GAP-43 is upregulated in Schwann cells of the injured motor axons (Vaudano et al., 1995), and physical activity including treadmill exercise not only increases Schwann cell 

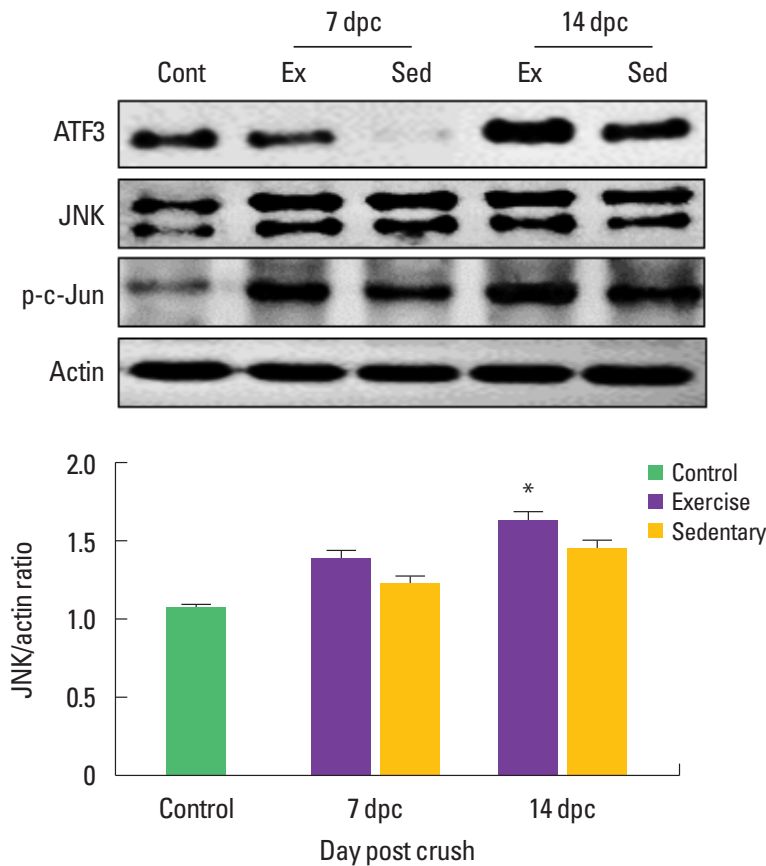
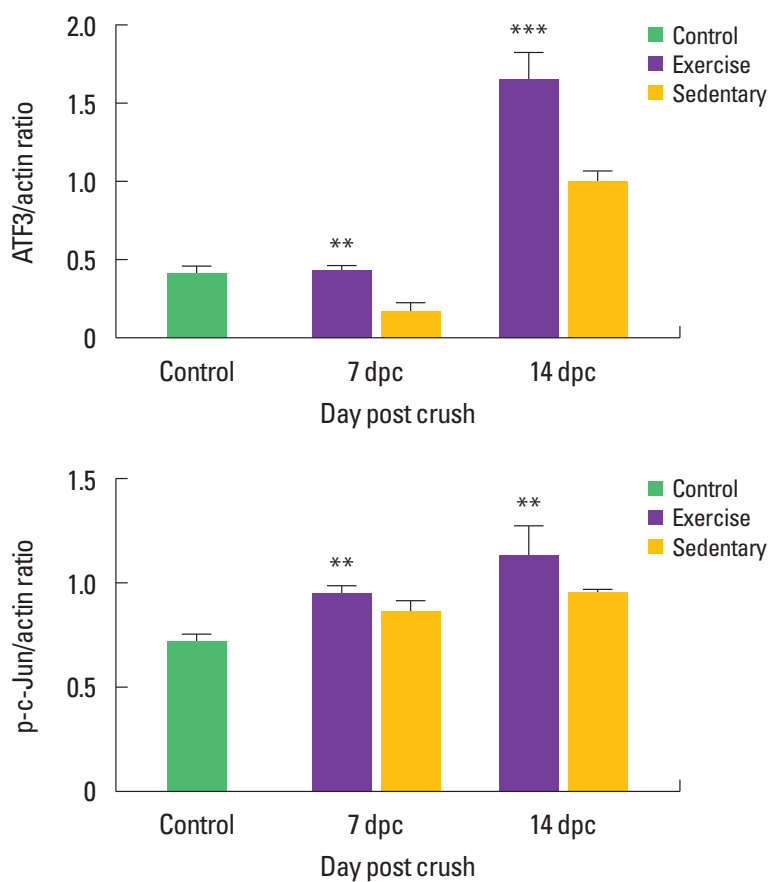

Fig. 3. Treadmill exercise-activated ATF3-mediated c-Jun phosphorylation at early stage of sciatic nerve regeneration. (A) ATF3 protein was increased in exercise group (Ex) compared to those in the sedentary group (Sed) at both 7 and $14 \mathrm{dpc}$. Treadmill exercise significantly upregulated JNK expression levels at $14 \mathrm{dpc}$. Also p-c-Jun was further increased in exercise group than those in the sedentary group (Sed). (B) The lower panel shows quantitation of western blot analysis. ${ }^{*} P<0.05$, ${ }^{* *} P<0.01,{ }^{* * *} P<0.001$ vs. the control group. dpc, days post crush; Cont, control; Ex, treadmill exercise; Sed, sedentary.

proliferation at early stage of sciatic nerve regeneration but also upregulates GAP-43 expression levels in proliferated Schwann cells (Udina et al., 2011a; Wilhelm et al., 2012), emphasizing the importance of the timing and duration of exercise for improving sciatic nerve regeneration. The results of these previous studies support our findings that treadmill exercise facilitated regrowth of the injured axons through activation of GAP-43 protein.

It has been known that GAP-43 activation is closely related with MAPKs signaling pathway secreted in regenerating nervous system (Cui et al., 2017; Fan et al., 2017). MAPKs, a family of serine/threonine specific kinases, are a signaling pathway to play a role in various cellular responses ranging from proliferation and differentiation to apoptosis, which is like a double-edged sword. MAPKs consist of three subfamilies such as ERK1/2, JNK, and p38 kinase. In the nerve regeneration field, Zhang et al. (2018) reported that ERK1/2 could regulate phosphorylation of CREB for promoting neurite outgrowth and cell survival in the damaged nerves. In our study, p-ERK1/2 was enhanced by treadmill exercise at only $7 \mathrm{dpc}$ and decreased to normal level $14 \mathrm{dpc}$. But its downstream molecules including JNK, c-Jun, and p-CREB showed a tendency to increase continuously until $14 \mathrm{dpc}$ by exercise. These data implicate that ERK1/2-regulated Schwann cell proliferation might be associated with axonal regrowth at early stage of regeneration. Seo et al. (2009) demonstrated that treadmill exercise is one of the most therapeutic tools for enhancing cell proliferation and axonal elongation of the injured sciatic nerve via phosphorylation of ERK1/2 protein. In addition, many researchers suggested that the other main MAPK signaling pathways including JNK and c-Jun (Barr and Bogoyevitch, 2001; Davis, 1994) as well as phosphorylation of CREB (Sabatier and English, 2015; Udina et al., 2011b) are activated by peripheral nerve injury and regular exercise after SNI further facilitates these proteins in the damaged Schwann cells compare to those in the sedentary group. Base on the biochemical similarity of MAPK and CREB in nerve regeneration after exercise, we consider that ERK1/2 and CREB signaling pathways contribute to axonal elongation with Schwann cell proliferation.

In addition to activation of MAPK pathway, ATF3 also is a regulator for inducing GAP-43 expression in the injured peripheral nerves (Abe and Cavalli, 2008; Allodi et al., 2012). In general, ATF3 is examined in neurons and Schwann cells after crush or transection of sciatic nerve in rats (Saito and Dahlin, 2008), and it is deeply related with JNK and c-Jun activation. Blom et al. (2014) noted that JNK inhibition by SP600125 and (D)-JNK1 dramati- 
cally down-regulated ATF3 induction in the injured neurons and JNK-dependent activation of c-Jun is one of a variety of cellular reactions in response to nerve damage, which mean delay of nerve regeneration in injured nerve. Our study observed increased ATF3 expression in exercise group at both 7 and $14 \mathrm{dpc}$ compared to the sedentary group, and ATF 3 showed the same pattern of expression in JNK and p-c-Jun proteins in present study. According to the previous studies, it has been suggested that a number of different transcription factors including ATF3, c-Jun, CREB, and Oct-6 were founded in achieving anatomical and functional regeneration after SNI (Patodia and Raivich, 2012), and ATF3 might mediate GAP-43 expression in the damaged Schwann cells. Furthermore, exercise for $1 \mathrm{hr}$ at $50 \%$ maximum oxygen uptake transiently increased ATF3 induction in skeletal muscle (Fernández-Verdejo et al., 2017) as well as in nerves (Nakagomi et al., 2003).

Given these findings reported in previous and present studies, physical activity such as treadmill exercise would be important in developing therapeutic strategies for facilitating regeneration-related molecules in the damaged sciatic nerve. In conclusion, the present finding on exercise-activated ATF3 and MAPK pathway during peripheral nerve regeneration should provide a more precise biochemical mechanism on axonal regeneration.

\section{CONFLICT OF INTEREST}

No potential conflict of interest relevant to this article was reported.

\section{ACKNOWLEDGMENTS}

This work was supported by the Ministry of Education of the Republic of Korea and the National Research Foundation of Korea (NRF- 2018S1A5A8028577).

\section{REFERENCES}

Abe N, Cavalli V. Nerve injury signaling. Curr Opin Neurobiol 2008;18: 276-283.

Allodi I, Udina E, Navarro X. Specificity of peripheral nerve regeneration: interactions at the axon level. Prog Neurobiol 2012;98:16-37.

Armada-da-Silva PA, Pereira C, Amado S, Veloso AP. Role of physical exercise for improving posttraumatic nerve regeneration. Int Rev Neurobiol 2013;109:125-149.

Arthur-Farraj PJ, Latouche M, Wilton DK, Quintes S, Chabrol E, Banerjee A, Woodhoo A, Jenkins B, Rahman M, Turmaine M, Wicher GK, Mit- ter R, Greensmith L, Behrens A, Raivich G, Mirsky R, Jessen KR. c-Jun reprograms Schwann cells of injured nerves to generate a repair cell essential for regeneration. Neuron 2012;75:633-647.

Barr RK, Bogoyevitch MA. The c-Jun N-terminal protein kinase family of mitogen-activated protein kinases (JNK MAPKs). Int J Biochem Cell Biol 2001;33:1047-1063.

Blom CL, Mårtensson LB, Dahlin LB. Nerve injury-induced c-Jun activation in Schwann cells is JNK independent. Biomed Res Int 2014;2014: 392971.

Chen CJ, Ou YC, Liao SL, Chen WY, Chen SY, Wu CW, Wang CC, Wang WY, Huang YS, Hsu SH. Transplantation of bone marrow stromal cells for peripheral nerve repair. Exp Neurol 2007;204:443-453.

Cui J, Cui C, Cui Y, Li R, Sheng H, Jiang X, Tian Y, Wang K, Gao J. Bone marrow mesenchymal stem cell transplantation increases GAP-43 Expression via ERK1/2 and PI3K/Akt pathways in intracerebral hemorrhage. Cell Physiol Biochem 2017;42:137-144.

Davis RJ. MAPKs: new JNK expands the group. Trends Biochem Sci 1994; 19:470-473.

English AW, Cucoranu D, Mulligan A, Sabatier M. Treadmill training enhances axon regeneration in injured mouse peripheral nerves without increased loss of topographic specificity. J Comp Neurol 2009;517:245255.

Fan L, Xiong Y, Fu Z, Xu D, Wang L, Chen Y, Xia H, Peng N, Ye S, Wang Y, Zhang L, Ye Q. Polyaniline promotes peripheral nerve regeneration by enhancement of the brain derived neurotrophic factor and ciliary neurotrophic factor expression and activation of the ERK1/2/MAPK signaling pathway. Mol Med Rep 2017;16:7534-7540.

Fernández-Verdejo R, Vanwynsberghe AM, Essaghir A, Demoulin JB, Hai T, Deldicque L, Francaux M. Activating transcription factor 3 attenuates chemokine and cytokine expression in mouse skeletal muscle after exercise and facilitates molecular adaptation to endurance training. FASEB J 2017;31:840-851.

Glenn TD, Talbot WS. Signals regulating myelination in peripheral nerves and the Schwann cell response to injury. Curr Opin Neurobiol. 2013; 23:1041-1048.

Gómez-Pinilla F, Ying Z, Roy RR, Molteni R, Edgerton VR. Voluntary exercise induces a BDNF-mediated mechanism that promotes neuroplasticity. J Neurophysiol 2002;88:2187-2195.

Gordon T. Electrical stimulation to enhance axon regeneration after peripheral nerve injuries in animal models and humans. Neurotherapeutics 2016;13:295-310.

Grinsell D, Keating CP. Peripheral nerve reconstruction after injury: a review of clinical and experimental therapies. Biomed Res Int 2014;2014: 698256.

Han IS, Seo TB, Kim KH, Yoon JH, Yoon SJ, Namgung U. Cdc2-mediated 
Schwann cell migration during peripheral nerve regeneration. J Cell Sci 2007;120(Pt 2):246-255.

Jiang X, Liu L, Zhang B, Lu Z, Qiao L, Feng X, Yu W. Effects of angelica extract on schwann cell proliferation and expressions of related proteins. Evid Based Complement Alternat Med 2017;2017:6358392.

Kalender AM, Dogan A, Bakan V, Yildiz H, Gokalp MA, Kalender M. Effect of Zofenopril on regeneration of sciatic nerve crush injury in a rat model. J Brachial Plex Peripher Nerve Inj 2009;4:6.

Ko KR, Lee J, Lee D, Nho B, Kim S. Hepatocyte growth factor (HGF) promotes peripheral nerve regeneration by activating repair Schwann cells. Sci Rep 2018;8:8316.

Martínez de Albornoz P, Delgado PJ, Forriol F, Maffulli N. Non-surgical therapies for peripheral nerve injury. Br Med Bull 2011;100:73-100.

Moore DL, Goldberg JL. Multiple transcription factor families regulate axon growth and regeneration. Dev Neurobiol 2011;71:1186-1211.

Nakagomi S, Suzuki Y, Namikawa K, Kiryu-Seo S, Kiyama H. Expression of the activating transcription factor 3 prevents c-Jun $\mathrm{N}$-terminal kinase-induced neuronal death by promoting heat shock protein $27 \mathrm{ex}$ pression and Akt activation. J Neurosci 2003;23:5187-5196.

Park JS, Höke A. Treadmill exercise induced functional recovery after peripheral nerve repair is associated with increased levels of neurotrophic factors. PLoS One 2014;9:e90245.

Patodia S, Raivich G. Role of transcription factors in peripheral nerve regeneration. Front Mol Neurosci 2012;5:8.

Sabatier MJ, English AW. Pathways mediating activity-induced enhancement of recovery from peripheral nerve injury. Exerc Sport Sci Rev 2015;43:163-171.

Saito H, Dahlin LB. Expression of ATF3 and axonal outgrowth are impaired after delayed nerve repair. BMC Neurosci 2008;9:88.

Seo TB, Han IS, Yoon JH, Seol IC, Kim YS, Jo HK, An JJ, Hong KE, Seo YB, Kim DH, Park SK, Yang DC, Namgung U. Growth-promoting activity of Hominis Placenta extract on regenerating sciatic nerve. Acta Pharmacol Sin 2006;27:50-58.

Seo TB, Oh MJ, You BG, Kwon KB, Chang IA, Yoon JH, Lee CY, Namgung U. ERK1/2-mediated Schwann cell proliferation in the regenerating sciatic nerve by treadmill training. J Neurotrauma 2009;26:1733-1744.

Teodori RM, Betini J, de Oliveira LS, Sobral LL, Takeda SY, de Lima Montebelo MI. Swimming exercise in the acute or late phase after sciatic nerve crush accelerates nerve regeneration. Neural Plast 2011;2011: 783901.

Udina E, Cobianchi S, Allodi I, Navarro X. Effects of activity-dependent strategies on regeneration and plasticity after peripheral nerve injuries. Ann Anat 2011a;193:347-353.

Udina E, Puigdemasa A, Navarro X. Passive and active exercise improve regeneration and muscle reinnervation after peripheral nerve injury in the rat. Muscle Nerve 2011b;43:500-509.

Vaudano E, Campbell G, Anderson PN, Davies AP, Woolhead C, Schreyer DJ, Lieberman AR. The effects of a lesion or a peripheral nerve graft on GAP-43 upregulation in the adult rat brain: an in situ hybridization and immunocytochemical study. J Neurosci 1995;15(5 Pt 1):3594-3611.

Wanner R, Gey M, Abaei A, Warnecke D, de Roy L, Dürselen L, Rasche V, Knöll B. Functional and molecular characterization of a novel traumatic peripheral nerve-muscle injury model. Neuromolecular Med 2017;19: 357-374.

Wilhelm JC, Xu M, Cucoranu D, Chmielewski S, Holmes T, Lau KS, Bassell GJ, English AW. Cooperative roles of BDNF expression in neurons and Schwann cells are modulated by exercise to facilitate nerve regeneration. J Neurosci 2012;32:5002-5009.

Zhang Z, Liu Y, Zhu X, Wei L, Zhu J, Shi K, Wang G, Pan L. Sciatic nerve leachate of cattle causes neuronal differentiation of PC12 cells via ERK1/2 signaling pathway. J Vet Sci 2018;19:512-518. 